\title{
Noise properties in Semiconductor Ring Lasers
}

\author{
Antonio Pérez S., Roberta Zambrini, Alessandro Scirè and Pere Colet \\ Instituto de Física Interdisciplinar y Sistemas Complejos, IFISC, CSIC-UIB, Campus UIB, \\ Ctra. Valldemossa km. 7.5, E-07122 Palma de Mallorca, Spain.
}

\begin{abstract}
We analyze a rate equation model in the Langevin formulation for the two modes of the electric field and the carrier density, modelling the spontaneous emission noise in a semiconductor ring laser biased in the bidirectional regime. We analytically investigate the influence of complex backscattering coefficient when the two modes are reinterpreted in terms of mode-intensity sum (I-Spectrum) and difference (D-spectrum). The D-spectrum represents the energy exchange between the two counterpropagating modes and it is shaped by the noisy precursor of a Hopf bifurcation influenced mainly by the conservative backscattering. The I-Spectrum reflects the energy exchange between the total field and the medium and behaves similarly to the standard relative intensity noise for single-mode semiconductor lasers. Good agreement between analytical approximation and numerical results is found.
\end{abstract}

Keywords: Semiconductor lasers, ring lasers, laser dynamics

\section{INTRODUCTION}

Semiconductor ring lasers (SRLs) ${ }^{1}$ gained interest due to their peculiar properties from both fundamental and applicative point of view. SRLs exhibit different operating regimes ranging from bidirectional-continuous wave regime, to a bidirectional with alternate oscillations regime, to a bistable regime, to mode locking and chaos. ${ }^{3-7}$ These variety of operating regimes makes them promising candidates for wavelength filtering, unidirectional travelling wave operation, and multiplexing/demultiplexing applications. In particular the bistable regime is interesting for applications in optical logics, optical gating and reshaping, ${ }^{8}$ whereas the bidirectional regime is also interesting for rotation sensing applications. ${ }^{9}$

From a fundamental point of view, the interaction of fluctuations and two-mode nonlinear system unveiled interesting physics and new phenomena, e.g. the phenomenon of stochastic resonance was demonstrated in a ring dye laser. ${ }^{10}$ Also, fluctuations are important in applications for ring lasers, as they determine the performance of the ring laser gyroscope, ${ }^{11}$ or induce spontaneous switching in a bistable SRL. ${ }^{12}$ The main noise source of a semiconductor laser is represented by spontaneous emission, which yields to fluctuations of the emitted intensity an frequency. ${ }^{13}$ Different examples of how to model the spontaneous emission noise are shown in. ${ }^{14-16}$ While SRL share some general characteristics with other kinds of ring lasers, they also have some distinctive features such as phase/amplitude coupling, which is known to enhance phase noise, ${ }^{13}$ and strong intermodal gain crossaturation, which induces anticorrelated dynamics in the mode-power distribution. ${ }^{3}$ Also, for technological reasons, SRLs experience conservative backscattering stronger than dissipative one as in gas or dye ring lasers. ${ }^{17}$ We address here how these features influence the noise spectra of SRL.

We consider the effects of the spontaneous emission noise in a two mode rate equation model, for a SRL operating in the bidirectional regime. We analytically calculate noise-spectra and correlations properties when the two modes are reinterpreted in terms of mode-intensity sum and difference taking into account the fluctuations on the phase of the fields. On one side the total intensity and carrier density show a noise spectrum (I-spectrum) characterized by a resonance induced by the typical field-medium exchange processes (relaxation oscillations) and the global phase invariance induced by the Goldstone mode, so as far as those variables are concerned, it behaves as a standard single-mode Fabry-Perot semiconductor laser. Besides, the degree of freedom associated to the simultaneous presence of two counterpropagating modes allows for a further process of energy exchange between the two modes. Our analysis unveiled that such process presents a resonance interpreted as a 'noisy precursor' of a Hopf bifurcation ${ }^{18}$ influenced mainly by the backscattering parameters.

Email: antonio@ifisc.uib.es 


\section{THE MODEL}

Considering a single longitudinal mode operation, the electric field inside the cavity reads

$$
E(x, t)=E_{+} e^{-i(\Omega t-k x)}+E_{-} e^{-i(\Omega t+k x)},
$$

where $E_{+}$and $E_{-}$are the mean-field slowly varying complex amplitudes of the electric field associated with the two propagation directions, $E_{+}$clockwise and $E_{-}$counterclockwise, respectively, being $x$ the spatial coordinate along the ring, assumed positive in the clockwise direction, and $\Omega$ is the optical frequency of the selected longitudinal mode. The model we consider is composed by the following set of dimensionless rate equations for the time evolution of the electric fields $E_{ \pm}$and the carrier density $N$

$$
\begin{aligned}
& \dot{E}_{ \pm}=\mathcal{G}_{ \pm}\left(N,\left|E_{ \pm}\right|^{2}\right) E_{ \pm}-\eta E_{\mp}+\xi_{ \pm}(t) \\
& \dot{N}=\gamma \mathcal{F}\left(N,\left|E_{ \pm}\right|^{2}\right)
\end{aligned}
$$

where

$$
\begin{gathered}
\mathcal{G}_{ \pm}\left(N,\left|E_{ \pm}\right|^{2}\right)=\frac{1}{2}(1+i \alpha)\left\{N \sigma_{ \pm}-1\right\}, \\
\mathcal{F}\left(N,\left|E_{ \pm}\right|^{2}\right)=\mu-N-N \sigma_{+}\left|E_{+}\right|^{2}-N \sigma_{-}\left|E_{-}\right|^{2}, \\
\sigma_{ \pm}=1-s\left|E_{ \pm}\right|^{2}-c\left|E_{\mp}\right|^{2},
\end{gathered}
$$

where $\eta=k_{d}+i k_{c}$ is the complex backscattering coefficient, with its dissipative $\left(k_{d}\right)$ and conservative $\left(k_{c}\right)$ components. The $\alpha$ factor describes the phase-amplitude coupling mechanism present in semiconductor lasers. The saturations effects in the gain, written in the quadratic approximation, are represented by $s$ and $c$, which are normalized self- and cross-gain saturation coefficients. The parameter $\mu$ is the pump parameter normalized to the threshold current (i.e. $\mu=1$ at threshold) and $\gamma$ is the ratio between the photon lifetime and the carrier lifetime. The model was proven to give excellent quantitative agreement with experiments. ${ }^{3,4}$ The fluctuations terms $\xi_{ \pm}(t)$ are the Langevin forces, ${ }^{20}$ i.e. white gaussian complex noise sources with the following non vanishing correlation properties

$$
\left\langle\xi_{ \pm}(t) \xi_{ \pm}^{*}\left(t^{\prime}\right)\right\rangle=2 \sqrt{\beta \tau_{p} N_{s t}} \delta\left(t-t^{\prime}\right),
$$

where $\tau_{p}$ is the photon lifetime, $N_{s t}$ is the carrier steady state solution (7) and $\beta$ represents the fraction of spontaneously emitted photons coupled to the cavity. Noise terms reflects the effect of spontaneous emission in each direction of propagation. For simplicity, we do not take into account a noise source for the carrier density equation, considering the spontaneous emission noise as the main noise source in semiconductor lasers. ${ }^{13,14}$

According to the experimental fitting ${ }^{3}$ through this paper we take the following parameters set (except where otherwise is noticed) $\alpha=3.5, s=0.005, c=0.01, k_{d}=3.2710^{-4}, k_{c}=4.410^{-3}, \tau_{p}=10 \mathrm{ps}, \gamma=210^{-3}$ and $\mu=1.2$.

\section{THEORETICAL ANALYSIS}

\subsection{Monochromatic symmetric solutions}

Neglecting the noise and by substituting in (2) the following monochromatic solution for the two fields with symmetric amplitude

$$
E_{ \pm}(t)=Q e^{i \omega t \pm i \phi}
$$

we find the stationary solutions. There are two possible cases, the in phase case

$$
\phi=0 \rightarrow \omega_{i n}=\alpha k_{d}-k_{c},
$$

and the out of phase case

$$
\phi=\frac{\pi}{2} \rightarrow \omega_{\text {out }}=-\alpha k_{d}+k_{c} .
$$

Depending of the sign of the backscattering parameters one of the solutions is stable and the other unstable, if $k_{d}>0\left(k_{d}<0\right)$ the out of phase case is stable (unstable). From now on we focus on the out of phase case, because we have chosen the parameters set in which such solution is stable, this can be shown without loosing 
generality. The corresponding stationary solution for the carrier density $N$ as a function of the amplitude of the fields and the pump parameter is

$$
N_{s t}=\frac{\mu}{1+2 Q^{2}\left(1-s Q^{2}-c Q^{2}\right)} .
$$

For the amplitude $Q$ we find

$$
Q^{2}=\frac{N_{s t}-1+k_{d}}{(c+s) N_{s t}}
$$

In the following subsection the linear stability of the stationary solution (6)-(8) is reported.

\subsection{Linear fluctuations dynamics}

Hereby we analyze the effect of a perturbation on the stationary solutions. We consider a real perturbation $n$ in the carrier density and complex perturbations $a_{ \pm}$for the fields

$$
\begin{gathered}
E_{ \pm}=\left(Q+a_{ \pm}\right) e^{i \omega t \pm i \phi}, \\
N=N_{s t}+n .
\end{gathered}
$$

By making use of (9) in (2) we derive the following linear system

$$
\begin{gathered}
\dot{n}=-\gamma\left\{n+\left[1-2 Q^{2}(s+c)\right] N_{s t} Q\left(a_{+}+a_{+}^{*}+a_{-}+a_{-}^{*}\right)+2 Q^{2}\left[1-s Q^{2}-c Q^{2}\right] n\right\} \\
\dot{a}_{ \pm}=\frac{1}{2}(1+i \alpha)\left\{N_{s t}\left(1-s Q^{2}-c Q^{2}\right) a_{ \pm}-N_{s t} Q^{2}\left[s\left(a_{ \pm}+a_{ \pm}^{*}\right)+c\left(a_{\mp}+a_{\mp}^{*}\right)\right]+Q\left(1-s Q^{2}-c Q^{2}\right) n-a_{ \pm}\right\} \\
-i \omega a_{ \pm}-\eta(\cos 2 \phi \mp i \sin 2 \phi) a_{\mp}+\xi_{ \pm} .
\end{gathered}
$$

At this point we introduce a new set of variables to simplify the set (10) in two independent problems by block diagonalization. The new variables are

$$
\begin{aligned}
& S(t)=a_{+}+a_{-}, \\
& R(t)=a_{+}-a_{-} .
\end{aligned}
$$

We can relate those new variables to experimentally accessible intensity variables $\left|E_{+}\right|^{2}$ and $\left|E_{-}\right|^{2}$ defining

$$
\begin{aligned}
& \mathbb{I}=\left|E_{+}\right|^{2}+\left|E_{-}\right|^{2}, \\
& \mathbb{D}=\left|E_{+}\right|^{2}-\left|E_{-}\right|^{2},
\end{aligned}
$$

and writing those new variables as $\mathbb{I}=I_{0}+I$ and $\mathbb{D}=D_{0}+D$, where $I_{0}$ and $D_{0}$ are constants and the perturbations $I$ and $D$ can be expressed in terms of $S$ and $R$ at first order,

$$
\begin{aligned}
& I=Q\left(S+S^{*}\right), \\
& D=Q\left(R+R^{*}\right) .
\end{aligned}
$$

The block involving $I$ describes a perturbations to the total laser intensity and it is coupled to the carrier density perturbation equation. The variable $I$ describes the perturbation of the total intensity of the lasers, regardless its distribution between the two modes. On the other side, $D$ describes the power exchange between the two counterpropagating fields.

\subsection{Relative Intensity}

The equation for the dynamic evolution of $R$ corresponding to the out of phase solution is

$$
\dot{R}=(1+i \alpha) K\left(R+R^{*}\right)-2 \eta R+\xi_{R}(t),
$$

where the fluctuation term is derived from (11) and $(3), \xi_{R}(t)=\xi_{+}(t)-\xi_{-}(t)$ with the correlation properties

$$
\left\langle\xi_{R}(t) \xi_{R}^{*}\left(t^{\prime}\right)\right\rangle=4 \sqrt{\beta \tau_{p} N_{s t}} \delta\left(t-t^{\prime}\right),
$$




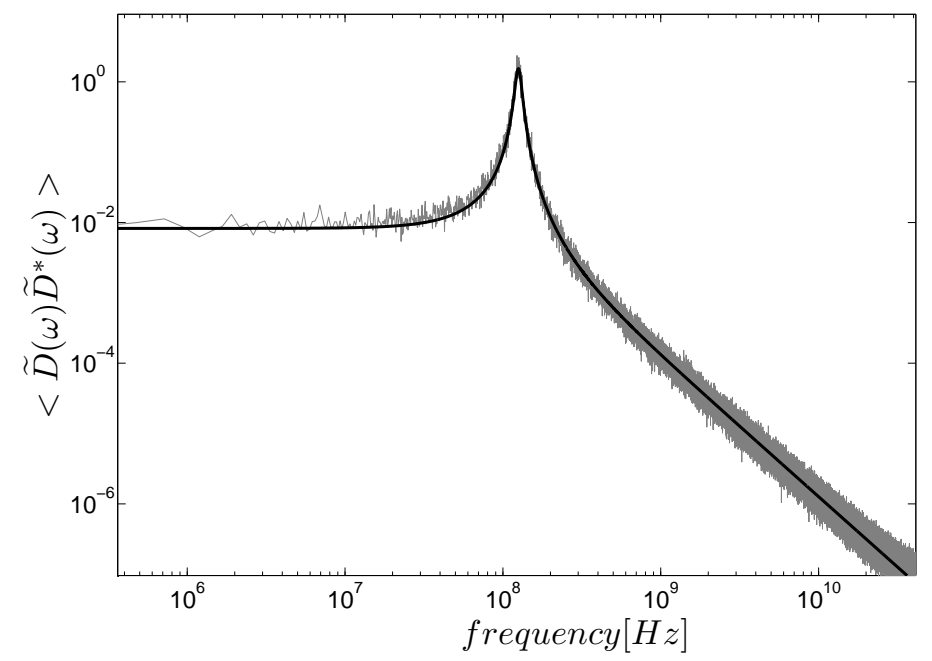

Figure 1. D-spectrum, the grey line corresponds to the numerical simulation for 20 realizations the analytical solution is the black line. $\beta=10^{-3} n s^{-1}, \alpha=3.5, s=0.005, c=0.01, k_{d}=3.2710^{-4}, k_{c}=4.410^{-3}, \gamma=210^{-3}$ and $\mu=1.2$.

and $K$ is a real constant defined by

$$
K=\frac{1}{2} N_{s t} Q^{2}(c-s)
$$

The corresponding eigenvalues for the differential equations system involving $R$ and $R^{*}$ are

$$
\lambda_{1,2}=K-2 k_{d} \pm\left[K^{2}+4 K \alpha k_{c}-4 k_{c}^{2}\right]^{\frac{1}{2}} .
$$

The analytical solution of the above system is

$$
\widetilde{R}(\omega)=\frac{1}{A(\omega)}\left[\left(i \omega-(1-i \alpha) K+2 \eta^{*}\right) \widetilde{\xi}_{R}(\omega)+(1+i \alpha) K \widetilde{\xi}_{R}^{*}(-\omega)\right]
$$

where

$$
A(\omega)=-\omega^{2}+i \omega\left(4 k_{d}-2 K\right)-4\left(k_{d}+\alpha k_{c}\right) K+4\left(k_{d}^{2}+k_{c}^{2}\right)
$$

Then we yield to the following analytical expression for the ensemble average

$$
\left\langle\widetilde{R}(\omega) \widetilde{R}^{*}\left(\omega^{\prime}\right)\right\rangle=\frac{1}{A(\omega) A(-\omega)}\left[4 k_{d}^{2}-4 K\left(k_{d}+k_{c} \alpha\right)+2 K^{2}\left(1+\alpha^{2}\right)+2 K \alpha \omega+\left(\omega-2 k_{c}\right)^{2}\right] 8 \pi \sqrt{\beta \tau_{p} N_{s t}} \delta\left(\omega-\omega^{\prime}\right) .
$$

Fig. 1 shows the D-spectrum, it is straightforward to demonstrate with (13) and (15) that

$$
<\widetilde{D}(\omega) \widetilde{D}^{*}\left(\omega^{\prime}\right)>=Q^{2}\left(<\widetilde{R}(\omega) \widetilde{R}^{*}\left(\omega^{\prime}\right)>+<\widetilde{R}(-\omega) \widetilde{R}^{*}\left(-\omega^{\prime}\right)>\right)
$$

spectrum at first order, using (21) and numerical simulations of the nonlinear system (2) using a second order Heun algorithm. ${ }^{14}$ Physically, the backscattering represents the energy exchange rate between the two modes. Such process shows a resonance (the peaks in Fig. 1).SRLs are well modeled by strong cross-saturation and conservative backscattering. For such parameters choice, our study unveils the presence of a resonance peak in the radiofrequency spectrum. This feature was reported in recent experimental works. ${ }^{21}$

\subsection{Total intensity and carrier density}

The equations for the dynamic evolution of $S$ and $n$ corresponding to the out of phase solution are

$$
\begin{gathered}
\dot{S}=(1+i \alpha)\left\{C n+\widetilde{K}\left(S+S^{*}\right)\right\}+\xi_{S}(t) \\
\dot{n}=-\gamma\left\{n+\left[1-2 Q^{2}(s+c)\right] N_{s t} Q\left(S+S^{*}\right)+2 Q^{2}\left[1-s Q^{2}-c Q^{2}\right] n\right\},
\end{gathered}
$$




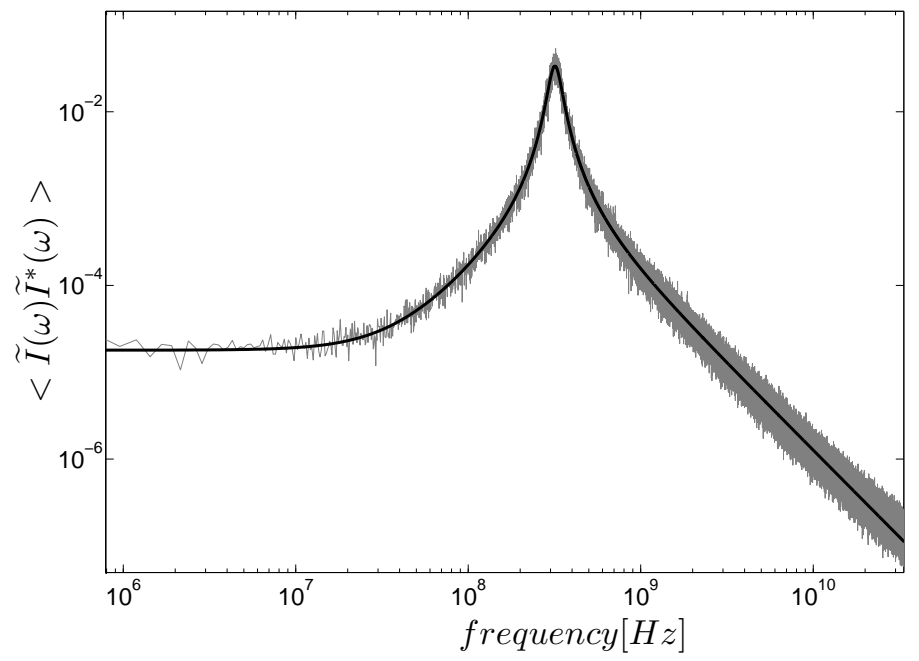

Figure 2. I-spectrum. $<\widetilde{I}(\omega) \widetilde{I}^{*}(\omega)>$ versus dimensionless frequency, $\omega$. The grey line corresponds to the numerical simulation and the black line is the analytical solution (31), $\beta=10^{-3} n s^{-1}$.

where the fluctuation term is derived from (11) and (3), $\xi_{S}(t)=\xi_{+}(t)+\xi_{-}(t)$ with the same correlation properties shown in the previous section (17) and $\widetilde{K}$ and $C$ are real constants

$$
\begin{aligned}
& \widetilde{K}=-\frac{1}{2} N_{s t} Q^{2}(c+s), \\
& C=Q\left(1-Q^{2}(c+s)\right) .
\end{aligned}
$$

The corresponding eigenvalues for the system (23) are

$$
\begin{gathered}
\lambda_{1,2}=0, \\
\widetilde{K}-\frac{\gamma}{2}-\gamma Q C \pm \frac{1}{2}\left[\gamma^{2}+4\left(\widetilde{K}^{2}+\widetilde{K} \gamma+\gamma^{2} Q C(1+Q C)\right)-8 \gamma\left(\widetilde{K} Q C+N_{s t} C^{2}\right)\right]^{\frac{1}{2}} .
\end{gathered}
$$

The presence of a zero eigenvalue indicates that the system (23) is singular. This fact is due to the presence of a Goldstone mode, which induces a global phase invariance. Indeed the Goldstone mode is associated to the imaginary part of $S$. Using (14) we get rid of the Goldstone mode by reducing the dynamics to a subspace orthogonal to the Goldstone mode. In terms of the variable $I$ the equations system (23) reads

$$
\begin{aligned}
\dot{I} & =2 \widetilde{K} I+2 Q C n+\xi_{I}(t) \\
\dot{n} & =-\gamma\left(1-2 Q^{2}(s+c)\right) N_{s t} I-\gamma(1+2 Q C) n,
\end{aligned}
$$

where the fluctuation term is derived from (11) and $(3), \xi_{I}(t)=Q \mathbb{R} e\left(\xi_{S}(t)+\xi_{S}^{*}(t)\right)$ with the correlation properties

$$
\left\langle\xi_{I}(t) \xi_{I}\left(t^{\prime}\right)\right\rangle=\left\langle\xi_{I}(t) \xi_{I}^{*}\left(t^{\prime}\right)\right\rangle=8 Q^{2} \sqrt{\beta \tau_{p} N_{s t}} \delta\left(t-t^{\prime}\right) .
$$

By Fourier transform, we derive

$$
\widetilde{I}(\omega)=\frac{1}{B(\omega)}[i \omega+\gamma(1+2 Q C)] \widetilde{\xi}_{I}(\omega)
$$

and

$$
\widetilde{n}(\omega)=\frac{-1}{B(\omega)} \gamma N_{s t}\left[1-2 Q^{2}(s+c)\right] \widetilde{\xi}_{I}(\omega),
$$

where

$$
B(\omega)=-\omega^{2}+i \omega[\gamma(1+2 Q C)-2 \widetilde{K}]+2 \gamma\left[Q C N_{s t}+\widetilde{K}(2 Q-1)\right]
$$




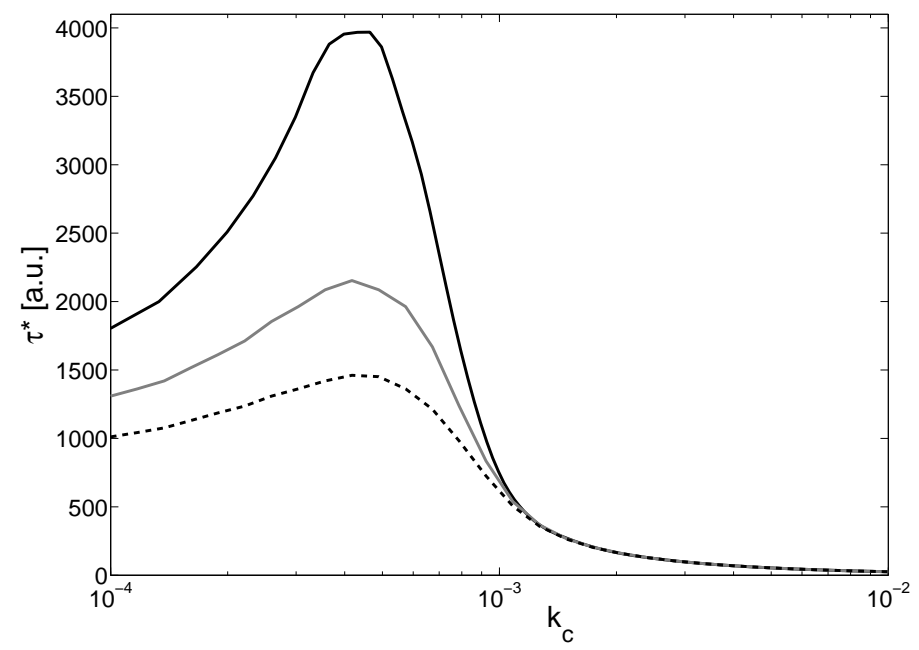

Figure 3. Decay time $\tau^{*}$ versus conservative backscattering coefficient $k_{c}$, the black line corresponds to $k_{d}=710^{-4}$. The grey to $k_{d}=810^{-4}$ and black dashed curve to $k_{d}=910^{-4}$.

we are able to find the following ensemble average

$$
<\widetilde{I}(\omega) \widetilde{I}^{*}\left(\omega^{\prime}\right)>=\frac{Q^{2}}{\widetilde{B}(\omega) \widetilde{B}^{*}(\omega)}\left[\omega^{2}+\gamma^{2}(1+2 Q C)^{2}\right] \times 16 \pi \sqrt{\beta \tau_{p} N_{s t}} \delta\left(\omega-\omega^{\prime}\right)
$$

Figure 2 shows the I-spectrum from (31) and from numerical simulations. The agreement between numerical and analytical solutions is very good.

\section{TIME CORRELATIONS}

We can relate the spectrum results of the previous sections to the auto-correlations of the variables $I(t)$ and $D(t)$, by the Wiener-Khinchin theorem. ${ }^{19}$

$$
\begin{gathered}
C_{D D}(\tau)=\frac{1}{2 \pi} \int_{-\infty}^{\infty}<D(\omega) D^{*}\left(\omega^{\prime}\right)>e^{i \omega \tau} d \omega \\
C_{I I}(\tau)=\frac{1}{2 \pi} \int_{-\infty}^{\infty}<I(\omega) I^{*}\left(\omega^{\prime}\right)>e^{i \omega \tau} d \omega
\end{gathered}
$$

The cross-correlation between $I(t)$ and $D(t)$ is zero due to the noise properties. Using (32) with (22) we calculate the correlation time for $D(t)$, the results are plotted in Fig. 3. Physically the conservative backscattering component makes the two modes uncorrelated.

\section{CONCLUSIONS}

We have studied the influence of spontaneous emission noise in a two-mode model for semiconductor ring lasers, biased in the bidirectional regime. The analysis has been carried out by linearizing the model close to a stable stationary solution, and considering effect of noise as stochastic perturbations expressed by Langevin forces. At a linear level, pertubations concerning the total intensity and carrier inversion dynamics decouple from the energy distribution processes between the two modes. This fact has permitted a full analytic analysis, well confirmed by numerical simulations of the complete non linear system. The analysis showed that semiconductor ring lasers have peculiar noise properties. On one side the total intensity and carrier density show a noise spectrum (I-spectrum) characterized by a resonance induced by the typical field-medium exchange processes (relaxation 
oscillations) and the global phase invariance induced by the Goldstone mode, so as far as those variables are concerned, it behaves as a standard single-mode Fabry-Perot semiconductor laser. Besides, the degree of freedom associated to the simultaneous presence of two counterpropagating modes allows for a further process of energy exchange between the two modes. Our analysis unveiled that such process presents a resonance influenced mainly by the backscattering parameters, and interpreted as a 'noisy precursor' of a Hopf bifurcation.

\section{ACKNOWLEDGMENTS}

This work was funded by the european project IOLOS IST-2005-34743 and Balear Government project QULMI PROGECIB-5A. A.S. and R.Z. acknowledge Ramón y Cajal program by Spanish MEC.

\section{REFERENCES}

1. A. S. H. Liao and S. Wang, "Semiconductor injetion lasers with circular resonator", Appl. Phys. Lett. 36, 10 (1980).

2. T. Krauss, P. J. R. Laybourn and J. S. Roberts, "CW operation of semiconductor ring lasers", Electron. Lett. 26, 2095 (1990).

3. M. Sorel, A. Scirè, G. Giuliani, R. Miglierina, S. Balle, P. J. R. Laybourn and S. Donati, "Alternate oscillations in semiconductor ring lasers", Opt. Lett. 27, 1992, (2002).

4. M. Sorel, G. Giuliani, A. Scirè, R. Miglieria, S. Donati and P. J. R. Laybourn, "Operating regimes og GaAs-AlGaAs semiconductor ring lasers: Experiment and model", IEEE J. Quantum Electron. 39, 1187 (2003).

5. M. Sorel, P. J. R. Laybourn, G. Guiliani and S. Donati, "Unidirectional bistability in semiconductor waveguide ring lasers", Appl. Phys. Lett. 80, 3051 (2002).

6. C. Born, M. Sorel and S. Yu, "Linear and Nonlinear Mode interactions in a Semiconductor Ring Laser", IEEE J. Quantum Electron. 41, 261, (2005).

7. H. Nakatsuka, S. Asaka, H. Itoh, K. Ikeda and M. Matsuoka, "Observational bifurcation to chaos in all-optical bistable system", Phys. Rev. Lett. 50, 109 (1983).

8. M. T. Hill, H. J. S. Dorren, T.de Vrie, X. J. M. Leijtens, J. H. den Besten, B. Smalbrugge, Y. S. Oei, H. Binsma, G. D. Khoe and M. K. Smit, "A fast low-power optical memory based on coupled micro-ring laser", Nature 432, 206 (2004).

9. A. Pérez S., A. Scirè, "Theoretical Analysis of a New Technique for Inertial Rotation Sensing Using a Semiconductor Ring Laser", submitted (2008).

10. G. Vemuri and R. Roy, "Stochastic resonance in a bistable ring laser", Phys. Rev. A 39, 4668 (1989).

11. W. W. Chow, J. Gea-Banacloche, L. M. Pedrotti, V. E. Sanders, W. Schleich and M. O. Scully, "The ring laser gyro", Rev. Mod. Phys. 57, 61 (1985).

12. T. Pérez, A. Scirè, G. Van der Sande, P. Colet, C.R. Mirasso, "Bistability and all-optical switching in semiconductor ring lasers", Opt. Express 15, 12941 (2007)

13. K. Petermann, Laser Diode Modulation and Noise, Kluwer Academic Publishers, pp. 152-214 (1988).

14. M. San Miguel and R. Toral, "Stochastic effects in physical systems" in Instabilities and nonequilibrium structures VI, E. Tirapegui, J. Martínez and R.Tiemann, Kluwer Academic Publisheres, pp. 35-130, (2000).

15. S. Balle, P. Colet and M. San Miguel, "Statistics for the transient response of a single-mode semiconductor laser gain switching“, Phys. Rev. A 43, 498 (1991).

16. S. Balle, F. de Pasquale, N.B. Abraham and M. San Miguel, "Statistics of the transient frequency modulation in the switch-on of a single-mode semiconductor laser", Phys. Rev. A 45, 1955 (1992).

17. C. Etrich, P. Mandel, R. Centeno, R.J.C. Spreeuw and J.P. Woerdmann, "Dynamics of a ring laser gyroscope with backscattering", Phys. Rev. A 46, 525 (1992).

18. K. Wiesenfeld, "Virtual Hopf phenomenon: A new precursor of period doubling bifurcations", Phys. Rev. A 32, 1744 (1985).

19. G. P. Agrawal, "Mode-partition noise and intensity correlation in a two-mode semiconductor laser", Phys. Rev. A 37, 2488 (1988). 
20. C. W. Gardiner, Handbook of Stochastic Methods for Physics, Chemistry and the Natural Sciences, $2^{\text {nd }}$ Edition, Springer-Verlag, Berlin, p.107 (1990).

21. C. Ji, M. F. Booth, A. T. Schremer and J. M. Ballantyne, "Characterizing relative intensity noise in InGaAsPInP triangular ring lasers", IEEE J. Quantum Electron. 41, 925 (2005). 